\title{
STATUS OF THE 36 MHZ LINAC CAVITIES FOR THE GSI HIGH CURRENT INJECTOR
}

\author{
H. Gaiser, K. Kaspar, U. Ratzinger, GSI, Darmstadt, Germany, \\ S. Minaev, MEPHI Moscow, B. Krietenstein, TU Darmstadt
}

\section{Abstract}

The new 1.4 MeV/u prestripper linac at GSI will provide intense ion beams to fill the synchrotron SIS to the space charge limit for all elements up to uranium. The design mass to charge ratio $\mathrm{A} / \mathrm{q}$ is 65 , the effective acceleration voltage of the $30 \mathrm{~m}$ long LINAC is up to $91 \mathrm{MV}$. The design beam current in emA is $0.25 \cdot \mathrm{A} / \mathrm{q}$, i.e. $15 \mathrm{~mA}$ of $\mathrm{U}^{4+}$.

This linac will replace the $27 \mathrm{MHz}$ Unilac Wideröe section which was designed for A/q-values below 24. All cavities of the new linac are operated in the $\mathrm{H}_{110}$-mode. The $9.2 \mathrm{~m}$ long IH-RFQ with an energy profile from $2.2 \mathrm{keV} / \mathrm{u}$ to $120 \mathrm{keV} / \mathrm{u}$ has been completed and $\mathrm{rf}$ tuned. RF power tests were performed successfully on 2 out of 10 tank modules. The drift tube linac consists of two 42 MV IH cavities. The 'Combined Zero Degree Structure" KONUS allows to accelerate the beam by a total number of 99 gaps with only 5 quadrupole triplet lenses for transverse focusing.

The experience from the construction and from the rf tuning of all cavities is reported as well as the measured quality and shunt impedance values. The running in of the new prestripper linac is scheduled from April to September 1999.

\section{INTRODUCTION}

The High Current Linac conceptual design [1] was guided by the aim to fill the synchrotron SIS18 up to the space charge limit. Multi-turn injection during $100 \mu \mathrm{s}$ has to provide $4 \cdot 10^{11}$ ions for light ions like $\mathrm{Ne}^{10+}$ and $4 \cdot 10^{10}$ ions for $\mathrm{U}^{73+}$ respectively. Taking into account the beam losses of up to $98.5 \%$ caused by two stripping processes as well as the present state of the art in ion source development, the new $36 \mathrm{MHz}$ front end linac has to provide the beam intensities and an $\mathrm{A} / \mathrm{q}$ range as mentioned above. Besides the high-current mode (up to $16 \frac{2}{3} \mathrm{~Hz}, 1 \mathrm{~ms}$ pulse train) the new front end linac additionally has to allow the continuation of the original Unilac operation mode $(50 \mathrm{~Hz}, 5 \mathrm{~ms}$ at $\mathrm{A} / \mathrm{q} \leq 26)$ for experiments in nuclear physics and materials research and with beam energies below or around the Coulomb barrier. The main RFQ $[2,3]$ as well as the short adapter-RFQ, called Superlens [4], are operated in the $\mathrm{H}_{110}$-mode. The DTL consists of two tanks in the $\mathrm{H}_{11(0)}$-mode: There is a gap voltage decrease at all tank end regions to optimize the shunt impedance. Additionally, tank $\mathrm{IH} 1$ has a ramped gap voltage distribution due to the pronounced energy increase by a factor of 6.2 along that structure.
Compared to existing ion linacs the A/q design value as well as the beam intensity of the new GSI-injector are outstanding and made a development of efficient cavities for low operating frequencies necessary. The choice of 36 $\mathrm{MHz}$ - one third of the succeeding Alvarez section - is a compromise which allows to reach the following aims:

- The RFQ current limit is high enough.

- The DTL tank diameter of around $2 \mathrm{~m}$ corresponds to the $108 \mathrm{MHz}$ Alvarez section and can be handled in the GSI galvanic copper plating shop as well as within the Unilac tunnel.

- The frequency can be kept constant along the new linac, which improves the beam quality and beam stability considerably according to LORASR beam dynamics simulations [1].

- The new accelerator is short enough to minimize the costs for a reconstruction of the Unilac tunnel.

The matching between RFQ und DTL is accomplished by a short low aperture quadrupole doublet at the RFQ exit followed by the 11 cell Superlens. The latter is operated at $-90^{\circ}$ and focuses the beam in 3-dim space into the DTL. This design avoids the local separation between the transverse focusing magnetic lens and a rebuncher.

So far the low level rf measurements and the tuning of all cavities were done successfully. A power test with 2 out of 10 RFQ sections was performed to check the performance of that new structure. The entire linac will be powered after the installation in the Unilac tunnel which is taking place at present.

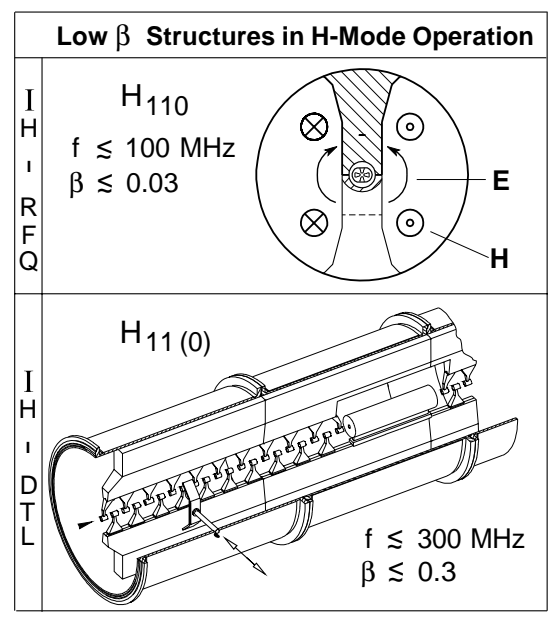

Figure 1: Cross sectional view of the IH-RFQ with electric and magnetic field directions and 3D-plot of the IH2cavity with the quadrupole triplet lens in the second module. 


\section{RFQ STRUCTURE}

The cavity design was developed at GSI during '94 and '95. At the end of '96 the 10 tank modules were ready for in-house copper plating. A first assembly of the whole RFQ with mini vanes took place in July '97 [5]. The resonant frequency was about $1 \%$ higher than predicted by MAFIA calculations [3]. Additionally, it was decided to use inductive tuning by cylindrical plungers instead of capacitive tuning for this cavity. Therefore a total frequency shift of $0.5 \mathrm{MHz}(-1.5 \%)$ had to be provided. The IH-RFQ contains 100 mini vane-carrier rings along the $9.2 \mathrm{~m}$ long structure. A remake of these rings with modified geometry allowed to distribute the needed capacity increase of $3 \%$ along the whole tank length. At the same time the junction principle was changed (Fig. 2).

In July '98 a RFQ power test was performed: The first and the last of the 10 RFQ modules were assembled to form a short $\mathrm{H}_{110}$-cavity. After a total operation time of about 10 days 4 goals were reached and partly exceeded:

- The vane-vane design voltage of $137 \mathrm{kV}$ was kept stable at duty factors of up to $1 \%$ and pulse lengths up to $1 \mathrm{~ms}$.

- The $40 \%$ design voltage level was held at a duty factor of $30 \%$.

- The resonant frequency and Q-value returned to their initial values in all cases after switching off the rf power.

- Reliable amplifier operation was proven and all control loops were activated successfully.

The power tests were also used to estimate the temperature increase of the electrode structure with carrier rings (Fig. 2). These parts are made from massive copper and are cooled via heat conduction by the water cooled stems only. As the temperature difference along the electrodes was calculated to remain below $0.4 \mathrm{~K}$ [2] it is assumed that the main effect is caused by temperature steps across the two bolted connections stem/carrier ring and carrier ring/electrode with $\Delta \mathrm{T}_{2} \approx 2 \cdot \Delta \mathrm{T}_{1} \cdot \Delta \mathrm{T}_{1}$ and $\Delta \mathrm{T}_{2}$ are the differences relatively to the stem temperature, the latter being approximately independent from the rf power level within the investigated range.

$\Delta \mathrm{T}_{2}$ was derived by measuring the resonance frequency shift and calculating the related capacity change between the electrodes due to heat expansion. The empirical formula from Ref. [6] was used to calculate the quadrupole capacity change caused by expansion of $\mathrm{d}$ and $\mathrm{h}$ (Fig. 2). This expansion results in a shortening of the RFQ parameters $\mathrm{R}_{0}$, a and $\rho$. For the $36 \mathrm{MHz}$ IH-RFQ the electrode capacity $C_{Q}^{\prime}$ is $108.5 \mathrm{pF} / \mathrm{m}$ while the total capacity is $184.5 \mathrm{pF} / \mathrm{m}$ [7].

Summary of Results from the Power Test:

A rf peak power level of $20 \mathrm{~kW}$ with $30 \%$ duty cycle into the $1 \& 10$-module array causes a resonance shift of $3 \mathrm{kHz}$. This corresponds to $\Delta C_{Q}^{\prime} / C_{Q}^{\prime}=3.5 \cdot 10^{-4}$.

With $\bar{h}=22.2 \mathrm{~mm}, \mathrm{~d}=11 \mathrm{~mm}$ and the relations

$$
\begin{aligned}
\mathrm{R}_{\mathrm{o}} / \rho(\Delta \mathrm{T}) & =\mathrm{R}_{\mathrm{o}} / \rho(\Delta \mathrm{T}=0) \cdot\left(1-1.1 \cdot 10^{-4} \Delta \mathrm{T}\right) \\
\mathrm{a}(\Delta \mathrm{T}) & =\mathrm{a}(\Delta \mathrm{T}=0) \cdot\left(1-0.94 \cdot 10^{-4} \Delta \mathrm{T}\right)
\end{aligned}
$$

the temperature difference finally results to

$$
\Delta \mathbf{T}_{2} \approx 3 \mathrm{~K} \text {. }
$$

As the design power level into the $1 \& 10$-module assembly is only $11 \mathrm{~kW}$ at $30 \%$ duty cycle this result can be accepted. For $\Delta \mathrm{T}_{2}$-values above $10 \mathrm{~K}$ the probability of transverse electrode misalignment in the order of $0.1 \mathrm{~mm}$ is estimated to be rather high already.

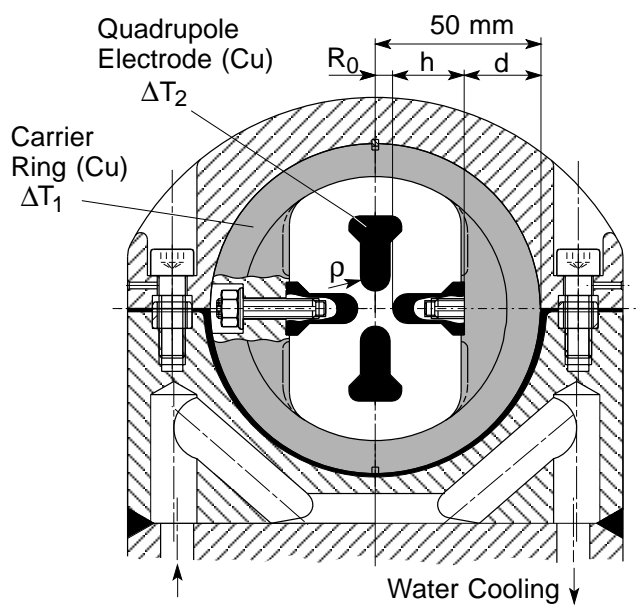

Figure 2: Mini vane electrodes with carrier ring mounted on a stem. The two bolted junctions will cause jumps in the temperature distribution under operation.

The final voltage distribution is flat and constant within $\pm 1 \%$ along the whole electrode length of $9.2 \mathrm{~m}$ and is independent of the plungers position which have a tuning range from $-0.2 \%$ to $+1 \%$ around the operating frequency (Fig. 3). The Q-value is 13000.

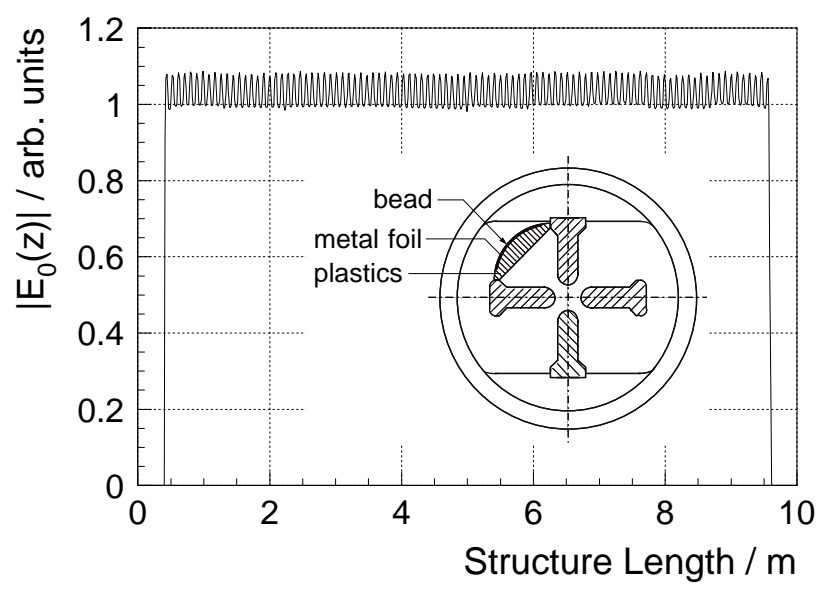

Figure 3: Measured vane-vane voltage along the $36 \mathrm{MHz}$ IH-RFQ. The fast oscillation is caused by field distortions from the mini vane carrier rings along the path of the bead. The small steps between modules are due to small changes in the vane-profile. 
At present the RFQ tank is being installed in the Unilac tunnel. Beam tests will be performed early in May '99.

\section{IH-DTL}

The design of the 83.2 MV DTL is described in ref. [1]. Each of the two IH-tanks consists of 4 modules and two endplates from black steel. The delivery to GSI lasted till to the end of ' 97 . The in house copper plating of these modules with an inner diameter of around $2 \mathrm{~m}$ and with the two girder profiles pointing towards the beam axis (Fig. 1) was not trivial. A platinum coated titanium grid was modelled along the inner tank surface in a distance of around $50 \mathrm{~mm}$. Such an electrode generates a homogeneous electric field distribution within the bath during the nickel and copper plating. Good results with that method were obtained when plating smaller components like the RFQ modules. In case of the DTL modules however, the organic bath additives decomposed rapidly during the copper plating process, which takes about 5 hours to get a $100 \mu \mathrm{m}$ layer. In case of 3 modules a time consuming polishing procedure of the plated surface became necessary because of that unpleasant effect. The DTL plating ended successfully in October' 98.

First rf measurements on IH1 were done in September '98. The frequency was $1.1 \%$ higher than expected [8]. Both the resonance frequency and the ramped gap voltage distribution were optimized by means of fixed tuning blocks at foreseen positions (Fig. 4).

It is concluded that the cavity design concept based on MAFIA calculations and on perturbation measurements on a 1:5.88 scaled rf model delivered correct parameters within tolerances of around $1 \%$ for the resonant frequency and of around $\pm 5 \%$ for the sum of gap voltages per drift tube section. After final rf tuning the voltage gain per section deviates by less than $\pm 0.5 \%$ now. This result is important to avoid phase errors of the bunch after passing through each of the long cavity internal triplet lenses. Only one iteration step of the voltage distribution during the rf model measurements was done to get an acceptable agreement between the effective gap voltage distribution as used originally in LORASR beam dynamics simulations and of the according results from tank measurements (Fig. 4). RF tuning of the IH2 cavity happened in March '99. The Q-values are 29000 for IH1 and 34000 for IH2 and do fit to the linac parameter list contained in ref. [1]. Vacuum tests on IH1 and IH2 were successful after repairing module 2 of $\mathrm{IH} 1$ and the end plate at the low energy end of tank $\mathrm{IH} 2$.

\section{Quadrupole Lenses}

The laminated quadrupole cores are based on $1 \mathrm{~mm}$ thick sheets of Vacoflux 50 to get pole tip fields of up to $1.3 \mathrm{~T}$.

The magnet field levels and tolerances are according to the specifications for the lenses delivered to GSI so far. The production of the magnets is seriously delayed and not all technical problems are solved yet.
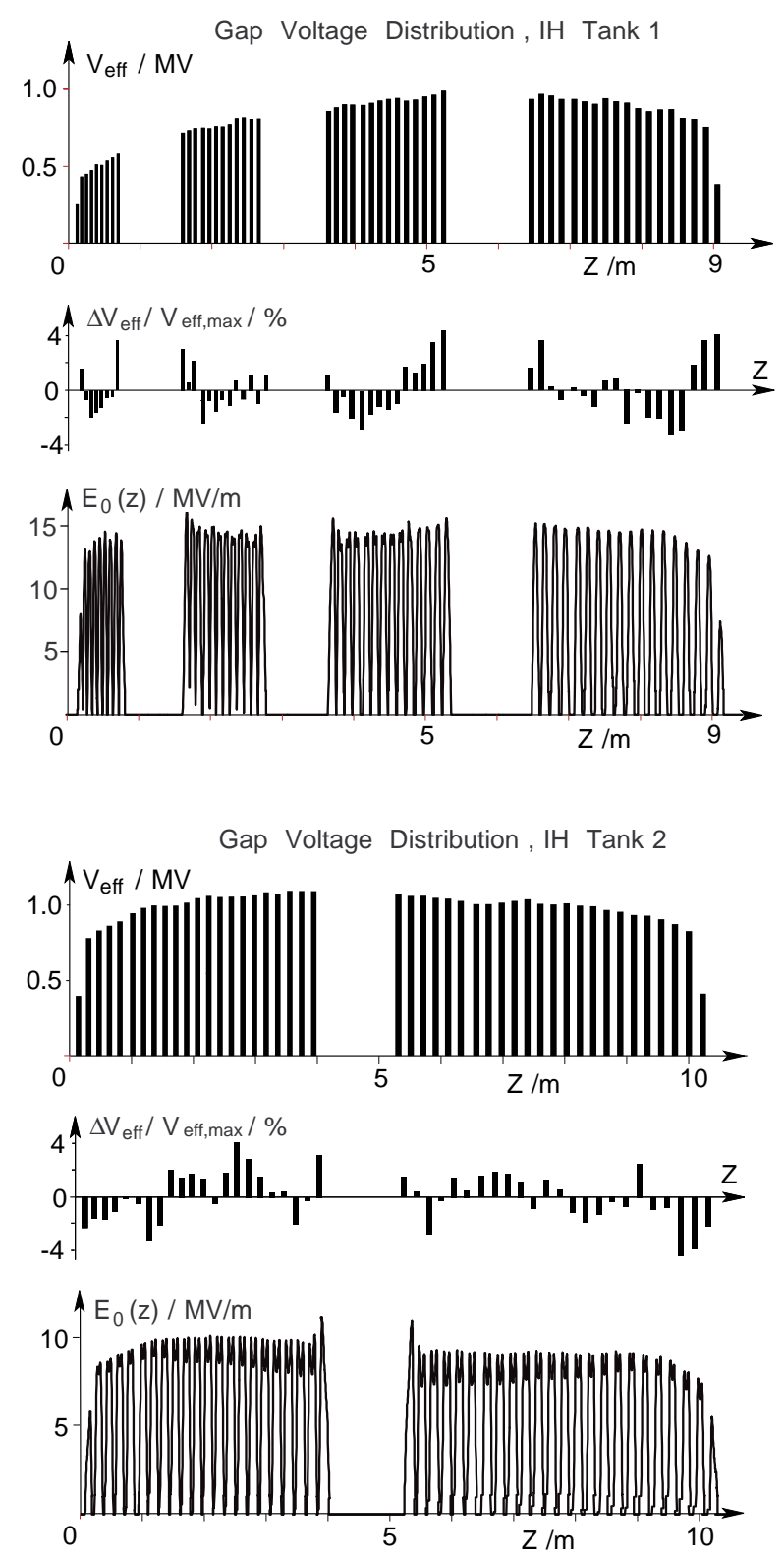

Figure 4: Effective gap voltage distribution as designed by LORASR-simulations, measured deviations of the tuned cavities and electric field distribution along the beam axis.

\section{REFERENCES}

[1] U. Ratzinger, Proc. LINAC 96, Geneva, CERN 96-07, p. 288.

[2] U. Ratzinger, K. Kaspar, E. Malwitz, R. Tiede, S. Minaev, Proc. EPAC 96, Barcelona, p. 304.

[3] K. Kaspar, U. Ratzinger, Proc. EPAC 96, Barcelona, p. 1973.

[4] U. Ratzinger, R. Tiede, Proc. LINAC 96, p. 128.

[5] U. Ratzinger, K. Kaspar, E. Malwitz, S. Minaev, R. Tiede, Nucl. Instr. and Meth. in Phys. Res., Sect. A 415, 281-286 (1998).

[6] I. Ben-Zvi, A. Jain, H. Wang, A. Lombardi, Proc. LINAC 90, Albuquerque, LA-12004-C, p. 73.

[7] U. Ratzinger, Habilitation, submitted at IAP, J.W. Goethe University, Frankfurt.

[8] B. Krietenstein, T. Weiland, U. Ratzinger, R. Tiede, S.A. Minaev, Proc. LINAC 96, Geneva, CERN 96-07, p. 243. 